\title{
TRANSICIÓN Y SENTIDO IDENTITARIO DE LA FORMACIÓN ACADÉMICA EN ESTUDIANTES SECUNDARIOS DE ORIGEN RURAL. UNA APROXIMACIÓN DESDE EL SUR DE CHILE
}

\section{Transition and identitary sense of academic formation in rural secondary students. An approach from the South of Chile}

\author{
ALEJANDRO ÁLVAREZ-ESPINOZA ${ }^{1}$ \\ DANIELA VERA-BACHMANN ${ }^{2}$ \\ PAULA ALMONACID GONZÁLEZ3 \\ MILLARAY CODERCH GARCÍA ${ }^{4}$ \\ FRANCISCA HAGEN HERNÁNDEZ ${ }^{5}$ \\ FRANCISCA PÜSCHEL CÁRDENAS ${ }^{6}$
}

Universidad Austral de Chile, Instituto de Psicología.

Correspondencia: aalvarez@spm.uach.cl; daniela.vera@uach.cl;

Recibido: 24-05- 2017

paula.almonacid.g@gmail.com; m.coderch.g@gmail.com;

Revisado: 08-07-2017

franciscahagenh@gmail.com; f.puschel.c@gmail.com

Aceptado: 10-12-2017

Resumen: Desde la tradición histórico-cultural, esta investigación explora los sentidos que otorgan a su actividad académica seis jóvenes de procedencia rural que cursan estudios secundarios en liceos urbanos de dos provincias del sur de Chile. Se utilizó la entrevista narrativa autobiográfica y el modelo de análisis de relatos de práctica propuesto por Gallardo y Sebastián (2012). Los resultados muestran la presencia de dos estructuras narrativas: heroica, en que un destinador impulsa al protagonista a obtener una recompensa, y de penitencia, donde el protagonista sufre temporalmente un destino indeseado. La primera se asocia a una articulación de sentido fuerte y alto nivel de agencia de los jóvenes respecto de su elección vocacional; la segunda, a una articulación de sentido débil y escaso nivel de agencia en la elección vocacional.

Palabras clave: sentido identitario; educación rural; educación secundaria; narrativa; psicología educacional.

\footnotetext{
${ }^{1}$ Magíster en Psicología, mención Psicología Educacional. Psicólogo. Académico de la Escuela de Psicología de la Universidad Austral de Chile-Sede Puerto Montt (Chile).

2 Doctora en Ciencias de la Educación. Psicóloga. Académica de la Escuela de Psicología de la Universidad Austral de Chile-Sede Puerto Montt (Chile).

${ }^{3}$ Licenciada en Psicología. Psicóloga. Universidad Austral de Chile-Sede Puerto Montt (Chile).

${ }^{4}$ Licenciada en Psicología. Psicóloga. Universidad Austral de Chile-Sede Puerto Montt (Chile).

${ }^{5}$ Licenciada en Psicología. Psicóloga. Universidad Austral de Chile-Sede Puerto Montt (Chile).

${ }^{6}$ Licenciada en Psicología. Psicóloga. Universidad Austral de Chile-Sede Puerto Montt (Chile).
} 
Abstract: From an historical-cultural approach, this research explores the perceptions towards academic activity from six rural students inserted in urban high schools in two provinces located in southern Chile. We used the autobiographical narrative interview and the model of analysis of practice stories proposed by Gallardo y Sebastián (2012). The results show the presence of two narrative structures: heroic, in which a destiner impels the protagonist to obtain a reward, and penitence, in which the protagonist temporarily suffers an undesired destiny. The first one is associated with a strong sense articulation and high level of agency of young people regarding their vocational choice; the second, with weak sense articulation and low level of agency in vocational choice.

Keywords: identity; rural education; secondary education; personal narratives; educational psychology.

\section{INTRODUCCIÓN}

La transición entre educación primaria y secundaria se ha señalado tradicionalmente en la literatura como un nudo crítico en las trayectorias educativas de los estudiantes (Ames y Rojas, 2011; Hernández y Raczynski, 2014; Raczynski, 2011; Rice, 2001; Tilleczek y Ferguson, 2007; West, Sweeting y Young, 2010). Parte importante de esta relevancia se relaciona con los notables cambios que supone tal transición, sus interacciones y sus consecuencias: desde las diferencias a nivel de organización de la enseñanza entre ambos sistemas $-\mathrm{y}$ los consecuentes cambios en el tipo de relaciones sociales que en estos espacios se promueven-, las transformaciones propias de la adolescencia y la pubertad que aparecen de forma relativamente simultánea a estos cambios, la influencia de largo plazo de la transición sobre las trayectorias educativas de los niños y jóvenes (Gimeno, 1997; West, Sweeting y Young, 2010), hasta el muy frecuente traslado a otro establecimiento e incluso a otras ciudades o pueblos en el caso de estudiantes de zonas rurales (Ames y Rojas, 2011). Todo ello puede expresarse en disminución de motivación o rendimiento académico, deserción o retraso escolar, baja autoestima y ansiedad social en los jóvenes, entre otros efectos (Tilleczek y Ferguson, 2007).

La importancia de esta transición en el contexto del ciclo vital de los jóvenes puede apreciarse además en su tradicional consideración como "rito de paso moderno" (Galton, 2009; Howard y Johnson, 2004), denominación que parece reconocer las fuertes implicancias identitarias que para los jóvenes representa el tránsito de primaria a secundaria, al menos en las sociedades occidentales. En efecto, en términos generales la transición educativa puede ser entendida como un momento o proceso críticos -en su sentido de riesgo y oportunidad- de cambio complejo, que experimenta el estudiante en su paso de un ambiente a otro en la búsqueda de nuevas oportunidades de desarrollo (Alvarado y Suárez, 2009). 
La mayor parte de las investigaciones enfocadas en la transición primariasecundaria se han realizado en grandes centros urbanos de países desarrollados, por lo cual es relativamente escasa la información respecto de la situación de países en vías de desarrollo. Existe además una ausencia notable de investigación en zonas rurales, ámbito en el cual se centran algunos estudios que han enfocado la transición a la enseñanza secundaria urbana por parte de jóvenes de origen rural en países como Australia, Finlandia y Canadá (Walsh, 1995; Pereira y Pooley, 2007; Pietarinen, 1998; Johnstone, 2002), siendo mucho más escasos en países en vías de desarrollo (Ames y Rojas, 2011; Hernández y Raczynski, 2014). En general, estos estudios muestran que jóvenes rurales y urbanos presentan similares expectativas y temores respecto de su tránsito a secundaria, aunque los primeros enfrentan dificultades específicas.

En Perú, el estudio de Ames y Rojas (2011) examina las transiciones a centros secundarios de niños urbanos y rurales de entre 11 y 13 años. De este último grupo, sus hallazgos destacan la fuerte valoración de la educación como forma de movilidad ascendente que hacen los jóvenes y sus familias; las transformaciones identitarias involucradas, a la vez que la presencia de dificultades que deben enfrentar, sobre todo en los períodos iniciales de la transición, como el temprano abandono de su hogar por la escasa oferta educativa local, la consecuente inserción en internados o lugares de acogida diferentes al hogar de origen y la negativa percepción de la calidad de la educación rural, implicando todo ello correlatos emocionales relevantes (Ames y Rojas, 2011).

En Chile, la investigación prácticamente no aborda este problema. De modo genérico, se sabe que las zonas rurales presentan tasas de reprobación y deserción escolar que son significativamente superiores a las de zonas urbanas (Ministerio de Educación de Chile, 2013b) y que la transición primaria-secundaria representa dinámicas de fracaso/abandono escolar que operan excluyendo particularmente a jóvenes de bajo estrato sociocultural y habitantes de zonas rurales (Ministerio de Educación de Chile, 2013a; Espínola, Claro y Walker, 2009; Santos, 2009).

Algunos trabajos han tematizado la relación entre niñez (Silva-Peña, Bastidas, Calfuqueo, Díaz y Valenzuela, 2013) o juventud rural (Rozas y Lara, 2009; Zapata, 2000) y educación, a través de la exploración de los significados que los estudiantes rurales le atribuyen, mostrando con claridad que la educación es concebida como una fundamental forma de movilidad social, permitiendo transitar desde el ámbito rural desvalorizado hacia un espacio urbano idealizado. El estudio de Hernández y Raczynski (2014) se enfoca específicamente en la transición de jóvenes de origen rural a establecimientos secundarios urbanos en la Región de la Araucanía. Sus conclusiones señalan que los jóvenes rurales mantienen representaciones positivas de la educación secundaria, implicando altas expectativas relacionadas con la posibilidad de abandonar el campo, lograr niveles de estudio superiores a sus padres y acceder a una profesión y trabajo en el espacio urbano; todas ellas alternativas vocacionales y laborales notablemente modeladas por la experiencia de su círculo cercano (amigos, 
familiares). Sin embargo, a la vez se presenta una importante brecha entre estas aspiraciones y las capacidades efectivas de los jóvenes y de sus familias para materializarlas, debido a factores económicos, desvalorización de la educación rural y ausencia de apoyo institucional e información.

Tales hallazgos resultan relevantes para la Región de Los Lagos, una de las regiones del país que presenta mayores índices de ruralidad (71,5\% de población rural), pobreza, e índices de abandono en educación secundaria, con predominio de la población rural (Ministerio de Educación de Chile, 2011; Instituto Nacional de Estadísticas de Chile, 2010). Tal situación se conjuga con fenómenos más amplios, como el aparente olvido de la educación rural por parte de la política educativa chilena (Leyton, 2013; Vera-Bachmann y Salvo, 2016) y abordajes investigativos de corte estadístico, centrados en el concepto de "deserción" (Ministerio de Educación de Chile, 2013a; Instituto Nacional de la Juventud de Chile, 2015) que, en general, tienden a no incluir la perspectiva de los jóvenes.

A ello debemos agregar un fenómeno central: la masificación de la educación secundaria en Latinoamérica, que plantea desafíos inéditos al sentido que este nivel educativo adquiere para la sociedad, familias, docentes y, particularmente, para los jóvenes. Desde esta perspectiva, la pregunta por el sentido de la educación para los estudiantes es relevante y ha sido destacada por autores como Tenti (2000) en Argentina, Baeza (2001), Gallardo (2012) y Valenzuela (2009) en Chile. En línea con lo anterior, la investigación destaca que un aspecto fundamental de estas transiciones y los cambios asociados a ellas es el compromiso identitario para los jóvenes, tanto por el momento del ciclo vital en que ocurren, como por el tipo de transformaciones involucradas.

En tal sentido, y de acuerdo con el planteo del enfoque de dinámicas identitarias (Bourgeois, 2009), las situaciones educativas activarían tensiones entre diversos aspectos del sí mismo (actual, deseado y normativo), a causa de que la incorporación a una actividad educativa evoca e interpela las expectativas sociales sobre el sujeto, las que pueden encontrarse alineadas -o en contraposición- respecto de las propias proyecciones, valores e identificaciones. Tales dinámicas producirían acciones de involucramiento o desinvolucramiento de los sujetos respecto del aprendizaje o de la situación educativa, susceptibles de ser interpretadas como estrategias identitarias (Gallardo y Sebastián, 2015). Lo expuesto hace relevante la pregunta acerca de qué sentidos construyen los jóvenes estudiantes de origen rural respecto a su educación en establecimientos urbanos de enseñanza media, que facilitarían o no su involucramiento y permanencia en el liceo. En el apartado siguiente se presentarán algunos elementos del marco conceptual que permite comprender el enfoque propuesto. 


\section{MARCO CONCEPTUAL}

\section{Educación rural en Chile}

En Chile existen 3.654 escuelas rurales, que atienden al 7,64\% del total de estudiantes del país (Elige Educar, 2016). En términos de sus resultados, la educación rural aún representa la modalidad y sector educacional más desaventajado, a pesar de los avances observados sobre todo durante la última década del siglo pasado (VeraBachmann y López, 2014). En general, los estudiantes de zonas rurales presentan una mayor tasa de deserción en comparación con los de zonas urbanas: $21,7 \%$ versus 13,9\% respectivamente (Ministerio de Educación de Chile, 2013b). Similar situación se aprecia respecto de las tasas de reprobación: las zonas rurales presentan índices comparativamente mayores, tanto en educación básica como en secundaria (Ministerio de Educación de Chile, 2013b; Vera-Bachmann, 2015).

La matrícula rural presenta tendencia al crecimiento hasta el $5^{\circ}$ grado de enseñanza primaria; en tanto a partir de $6^{\circ}$ grado comienza a disminuir (Ministerio de Educación de Chile, 2013b), fenómeno que puede ser razonablemente atribuido a motivos tales como que en este nivel los niños y niñas llegan a una edad que les permite trasladarse a poblados o ciudades cercanas para asistir a escuelas completas o liceos (Vera-Bachmann y Salvo, 2016). Efectivamente, los fenómenos migratorios han impactado negativamente en la matrícula de muchas escuelas rurales, determinando una activa política ministerial de cierre de escuelas, justificada desde criterios económicos, pero que desconoce los impactos psicosociales de tales medidas (Núñez, Solís y Soto, 2014).

\section{Sentido de la experiencia escolar}

Hasta los años 80 del siglo pasado, la investigación educacional prácticamente omitió la subjetividad o la construcción de la experiencia escolar por parte de los alumnos (Baeza, 2001). Actualmente, las transformaciones socioculturales y económicas, así como de la escuela como institución exigen del estudiante construir un sentido de la escolarización —que ya no es obvio ni dado-, lo que implica responder a la pregunta “¿para qué ir a la escuela?" (Tenti, 2000).

Este autor identifica tres disposiciones ideales que representan posibles respuestas por parte de los jóvenes secundarios latinoamericanos: a) la obligación, entendida como la obligación social de asistir a la escuela, donde no cabe elección posible; b) la razón instrumental, que implica una lógica de postergación de gratificaciones inmediatas por la expectativa de obtener mayores beneficios a futuro, pero donde la actividad académica en sí no tiene sentido; y c) el amor al conocimiento, que se relaciona a la entrega incondicional al saber entendido como valor en sí mismo. 
De acuerdo con este planteamiento, es más probable que aquellos grupos excluidos presenten la obligación/imposición como sentido de la experiencia escolar, en comparación con las clases medias, cuyas condiciones de vida y vivencia del tiempo, por ejemplo, hacen más probable la aparición de un sentido instrumental o incluso de amor al conocimiento. En similar dirección, Terigi (2009) propone una tipología que presenta las diversas formas en que se manifiesta la exclusión educativa, desde el no estar en la escuela y abandonarla, hasta formas más sutiles, como la escolaridad de baja intensidad, los aprendizajes elitistas/sectarios o los de baja relevancia.

En Chile, Valenzuela (2009) reconoce cinco tipos de motivos que darían sentido al aprendizaje escolar: ascenso social, desarrollo personal, responsabilidad social, sobrevivencia y mal menor. Empleando estas categorías, Silva-Peña et al. (2013) encontraron que el principal sentido de la educación expresado por niños y niñas mapuche rurales eran ascenso social y mal menor. Por su parte, Baeza (2001) —en su trabajo con jóvenes secundarios populares urbanos de la ciudad de Santiago - afirma que el liceo constituye una experiencia importante para los jóvenes populares chilenos, que lo representan como una "frontera" entre el discurso social de integración y la realidad cotidiana, donde el significado de la experiencia escolar se presentaría como un continuum desde la aceptación acrítica hasta el rechazo absoluto de la institución escolar.

Trabajando con similar población, y enfocado en el problema del involucramiento y del sentido identitario de la formación, desde una perspectiva histórico-cultural, Gallardo (2012) identificó tres estructuras narrativas: heroica, de conversión y de penitencia. Aquellos estudiantes con fuerte sentido identitario e involucramiento con el aprendizaje tendían a construir sus relatos conforme una estructura heroica, en la que un "destinador" establece un contrato con el protagonista del relato designándolo como héroe, quien ingresa al espacio heroico (espacio de formación) para realizar una serie de acciones —o pruebas - en función de alcanzar el objeto de búsqueda, hecho que es testimoniado por la obtención de una recompensa (Greimas, 1966; 1989).

En tanto, aquellos en que ese sentido-involucramiento era más débil, tendían a construir sus relatos empleando estructuras narrativas de conversión o penitencia. La primera de estas permite al sujeto referir los errores propios como parte de un pasado rechazado y radicalmente diferente al momento presente; en tanto en la segunda el protagonista se narra a sí mismo como sufriendo una muerte simbólica, siendo juzgado y enviado a un lugar indeseado en que cumplirá penitencia hasta expiar sus faltas (purgatorio), tras lo cual podrá ser conducido a un espacio ideal (paraíso) (Gallardo, 2012). El enfoque teórico de este último estudio corresponde al de la presente investigación, por lo cual se presentarán sintéticamente sus principales aspectos. 


\section{Enfoque de sentido identitario de la formación}

De acuerdo con Sebastián, Gallardo y Calderón (2016), es posible caracterizar el enfoque de sentido identitario de la formación a través de los siguientes aspectos:

- A mayor sentido personal que los participantes de una situación educativa experimenten a raíz de ella, mayor será la tendencia a que se involucren profundamente en las acciones que tal situación ofrece, incrementando con ello su probabilidad de aprender.

- El concepto de "sentido" empleado en este enfoque deriva de la teoría de la actividad de Leontiev (1984), contexto en el cual el término adquiere un significado preciso. Según esta teoría, la comprensión de los procesos psicológicos exige adoptar una perspectiva que reconozca que la actividad humana está estructurada por la anticipación de los efectos perseguidos por ella.

- Leontiev (1984) propone un esquema jerárquico de tres niveles estrechamente relacionados: actividades, acciones y operaciones. El nivel más amplio actividades- es guiado y definido a partir de un móvil, esto es, aquello que incitaría a un sujeto a actuar. A su vez, cada actividad particular se materializará en el nivel de las acciones - de menor alcance en términos temporales y espaciales, que se distinguirían entre sí en función de las metas que las orientan, es decir, de las representaciones de resultado esperado tras su realización. Por último, las acciones se llevarían a cabo mediante operaciones materiales concretas, bajo la forma de medios o procedimientos (Glassman, 1996).

- La teoría de la actividad propone una relación histórica y no mecánica entre estos niveles, de modo que diversas acciones pueden ser materializadas a través de un mismo procedimiento, así como distintas actividades pueden hacerlo a través de una acción determinada. A la vez, varios procedimientos pueden materializar una acción, así como diferentes acciones pueden materializar una actividad.

- Sebastián y De Villers (2006) proponen dos variantes en el modelo de Leontiev (1984): introducen el plano de los significados, a través de la incorporación del concepto de acción mediada de Wertsch (1993), lo cual permite ubicar más claramente el concepto de sentido en la tradición vygotskiana y, segundo, proponen concebir la identidad como una actividad humana orientada por el móvil permanente de producir una identidad, entendida esta como una experiencia de articulación narrativa de las sucesivas tomas de posición valorativas del sujeto (Penuel y Wertsch, 1995).

- Para Leontiev (1984), el sentido representa el grado de concordancia entre la meta de una acción y el móvil o motivo de la actividad a la cual pertenece. A mayor concordancia entre metas y motivo, mayor será el sentido que cobrarán las acciones (Sebastián y De Villers, 2006; Glassman, 1996). Por lo tanto, el concepto 
de sentido identitario de la formación refiere al grado de articulación entre las metas propias del espacio educativo y el motivo identitario, tal como es experimentado por el sujeto (Sebastián, Gallardo y Calderón, 2016), resultando de una elaboración individual, construida a partir de herramientas que se encontrarían en los significados sociales presentes en su comunidad.

Desde esta perspectiva, un estudiante se involucrará en una actividad educativa en tanto la perciba como coherente en términos de sentido respecto de su sí mismo; esto es, si tiene la posibilidad de vincular significativamente la actividad escolar con la propia identidad. Tal vinculación sería esencialmente narrativa (Arciero, 2003) e incidiría directamente en el involucramiento con su propio aprendizaje, entendido como la inversión cognitiva, la motivación puesta en la tarea y los afectos ligados con el aprendizaje por parte del estudiante.

Se han distinguido estos diversos grados de coherencia a partir de las tres respuestas que Tenti (2000) propone ante la pregunta "¿para qué ir a la escuela?". Estas respuestas sugieren tres niveles de posible articulación entre acción de formación y actividad identitaria, que se presentan en el Cuadro 1:

\section{Cuadro 1. Grados de articulación de sentido identitario en función de categorías de sentido de la actividad escolar}

\begin{tabular}{ll}
\hline Sentido & Grado de articulación \\
\hline \hline Obligación & Débil \\
Razón instrumental & Medio \\
Amor al conocimiento & Fuerte \\
\hline
\end{tabular}

Fuente: Tenti (2000)

\section{METODOLOGÍA}

Se trabajó desde un modelo metodológico cualitativo (Flick, 2004), con un diseño descriptivo-interpretativo (Eisenhardt, 1989; Yin, 1994). Participaron de la investigación seis jóvenes estudiantes - tres mujeres y tres varones, de entre 13 y 16 años- de procedencia rural, que cursan de $1^{\circ}$ a $3^{\circ}$ año de enseñanza secundaria en tres liceos urbanos: dos de dependencia municipal (de enseñanza técnico-profesional y científicohumanista) y uno particular subvencionado (técnico agrícola), de las ciudades de Puerto Montt y Osorno. 
Cuadro 2. Descripción de participantes según sexo, tipo de establecimiento (dependencia, modalidad y grupo socioeconómico del centro) y tiempo de iniciada la transición

\begin{tabular}{ccccc}
\hline Participante & Sexo & $\begin{array}{c}\text { Tipo de } \\
\text { establecimiento* }\end{array}$ & $\begin{array}{c}\text { GSE } \\
\text { establecimiento }\end{array}$ & $\begin{array}{c}\text { Tiempo inicio de } \\
\text { transición (años) }\end{array}$ \\
\hline \hline Z & Femenino & Municipal, HC & B & 0,5 \\
C & Femenino & Municipal, TP & A & 3,0 \\
D & Masculino & P. Subv., TP & B & 1,0 \\
S & Femenino & Municipal, HC & B & 0,5 \\
J & Masculino & P. Subv., TP & B & 0,5 \\
H & Masculino & Municipal, TP & A & 2,5 \\
\hline
\end{tabular}

«En función de tipo de dependencia (municipal o público; particular subvencionado y particular) y modalidad (HC: científico humanista; TP: técnico profesional). No se consideró la dependencia particular por razones asociadas a los criterios de inclusión/exclusión.

**Grupo socioeconómico del establecimiento: es determinado a través de la ponderación de las variables nivel educacional de ambos padres, ingreso total mensual del hogar e índice de vulnerabilidad (IVE). Categorías: bajo (A), medio bajo (B), medio (C), medio alto (D) y alto (E) (Agencia de Calidad de la Educación, 2012).

Fuente: Elaboración propia (2017)

La selección de los establecimientos educativos se realizó a través de muestreo intencionado (Patton, 1991), en función de criterios específicos de inclusión: establecimientos educacionales de las ciudades de Osorno y Puerto Montt, de dependencia municipal y particular subvencionada, pertenecientes a grupos socioeconómicos (GSE) bajo y medio bajo, urbanos, que reciban estudiantes provenientes de zonas rurales. Las bases de datos ministeriales no incorporan información respecto de este último criterio, sin embargo, la experiencia y conocimiento de terreno fueron críticos para el equipo en la identificación de estos establecimientos secundarios. Para seleccionar a los participantes se solicitó a los profesores jefes de cada establecimiento reportar a través de una ficha breve a sus estudiantes de origen rural: sector de procedencia, porcentaje de asistencia, rendimiento y observaciones generales. A partir de esos antecedentes se seleccionaron al azar seis participantes, atendiendo la paridad de género y la voluntariedad.

\section{Estrategia de producción de datos}

La técnica de recolección de información que se empleó fue la entrevista narrativa autobiográfica, acotada a la experiencia escolar (Bolívar, Domingo y Fernández, 1998), instrumento por medio del cual se obtuvo una serie de relatos de práctica, esto es, producciones narrativas específicas elaboradas por un sujeto respecto a su vida en tanto miembro participante de una práctica social concreta. Cada una de las entrevistas realizadas fue guiada por la misma consigna inicial: "Por favor, cuéntame como si fuera un cuento tu recorrido educativo". Las preguntas siguientes fueron orientadas en base a las respuestas de los entrevistados. Las entrevistas tuvieron una duración de entre 60 
y 95 minutos, siendo registradas en audio digital. Se realizó transcripción literal, intentando conservar fielmente las expresiones de los participantes, cuyos nombres se reemplazaron por letras para referirlos en el contexto de este estudio. Para cada uno de ellos se solicitó la aceptación voluntaria de un asentimiento informado, además del consentimiento informado dirigido a los padres, a fin de resguardar las condiciones de participación de cada uno de los estudiantes entrevistados.

\section{Estrategia de análisis}

El análisis se basó en la propuesta para la interpretación de relatos de práctica desarrollada por Gallardo y Sebastián (2012), inspirados en una adaptación del modelo actancial de Greimas (1966; 1989), realizada por Sebastián y De Villers (2006). Esta forma de análisis contempla cuatro fases, de las cuales las tres primeras corresponden a un análisis intracaso - que trata cada relato individualmente-, en tanto la última fase contempla análisis intercaso o transversal.

La fase 1 incluye las tareas de recogida de datos y transcripción, además de la reconstrucción temporal del relato, permitiendo obtener una síntesis cronológica de la experiencia relatada.

La fase 2 consiste en la exploración y categorización de elementos presentes en cada relato a partir de una grilla de análisis (Sebastián y De Villers, 2006) que permite conformar unidades textuales, caracterizadas porque presentan un vínculo entre dos eventos, entre un evento y una representación producida por el entrevistado, o bien entre dos representaciones, relevando aquellos vínculos reiterativos. El último procedimiento de esta fase se denomina puesta en relato, e implica la escritura por parte de los investigadores del relato recibido, que expresa la comprensión del analista sobre la narración analizada.

La fase 3 corresponde a la elaboración del análisis actancial del relato, que se propone identificar elementos —recursos materiales o simbólicos - que cumplan una función en el relato en tanto actantes, es decir, "elementos que ayudan o dificultan la trayectoria del protagonista o que deben ser alcanzados -o rechazados - por él para alcanzar su destino deseado" (Gallardo, 2012, p.53). La síntesis sincrónica implicada en este análisis es complementada por la diacronía de las cadenas de acciones medios-fines producto de la fase anterior.

La fase 4 corresponde a la generación de un análisis transversal intercasos, con el producto obtenido del análisis individual de cada relato analizado en las etapas precedentes, el cual permite la comparación a partir de las estructuras obtenidas de cada uno. 


\section{RESULTADOS}

A continuación, se presentan los resultados del análisis de los relatos de los participantes desde dos perspectivas complementarias: a) estructura narrativa y b) articulación de sentido identitario. De modo general, fue posible identificar dos estructuras narrativas, aparejadas a diversas modalidades de articulación de sentido identitario. Cuatro de los relatos comparten la estructura general de los cuentos maravillosos estudiados por Greimas (1989), también denominada "heroica" o "canónica", asociada a articulaciones de sentido identitario fuerte. Los dos relatos restantes se asimilan a una estructura que en otro estudio se ha denominado "de penitencia" (Gallardo, 2012), presentándose en ambos casos articulación de sentido identitario débil.

\section{Análisis de los relatos desde su estructura narrativa}

"Te prometo que voy a ser profesional":

Estructura narrativa heroica o canónica

En esta estructura narrativa un destinador envía al héroe al espacio heroico, donde debe superar diversas pruebas para obtener una recompensa (Gallardo, 2012), siendo apreciable en los relatos de Z, D, C y S. En el caso de S, tal figura corresponde a su abuela fallecida, con quien se ha comprometido a llegar a ser profesional, lo que constituye su objeto de búsqueda:

La última vez que hablé con mi abuelita... antes que fallezca... yo le prometí... que iba a llegar a ser profesional (...) le decía "te prometo que voy a ser profesional" (...) qué me diría si estuviera viva... no sé si estaría decepcionada de mí o me daría más fuerza para seguir adelante (S, mujer, Municipal HC).

Entre las pruebas que $S$ debe sortear en ese propósito, destaca el haber obtenido el primer lugar en el establecimiento donde cursó sus últimos años de enseñanza básica, a pesar de "ser de campo" como ella lo refiere y a las escasas expectativas de sus profesores debido a su origen rural:

Yo sentía eso, como que yo sabía menos, y en realidad no era así porque ese año yo tuve el primer lugar en el colegio en general, y al final yo le tapé la boca a todos profesores, inspectores, directores, porque ellos creían eso (S, mujer, Municipal HC).

En tanto, lograr ser la primera profesional de la familia se constituiría en la prueba definitiva o glorificante: ${ }^{7}$

\footnotetext{
7 En la estructura narrativa al modo de los cuentos maravillosos, Greimas (1966) distinguió tres pruebas a ser
} resueltas por el héroe: la prueba calificante, en que el héroe adquiere las capacidades necesarias para enfrentar la 
Llegar a ser una profesional (...) sería la primera de la familia, por parte de mi mamá, porque nadie ha llegado a ser profesional, o sea... nadie ha sacado una carrera (...) (S, mujer, Municipal HC).

Algo similar ocurre en los relatos de $Z$ y $C$. En ambos casos el destinador corresponde a la madre, a quien el héroe ofrece el objeto de búsqueda: la obtención de un título profesional:

Para que mi mamá no pague todo (...) no quiero seguir siendo una carga más ( $C$, mujer, Municipal TP).

Ella quería que nosotras hagamos exactamente lo mismo que mi hermana, surgir en este liceo y que tengamos un título profesional ( $Z$, mujer, Municipal HC).

En el caso de $\mathrm{D}$ el destinador es el mismo narrador, siendo su objeto de búsqueda el lograr administrar el campo familiar:

Esa es mi meta... y... así manejar un poco el tema del rubro con los animales, enfermedades... el control lechero, todas esas cosas, de a poquito ir realizándome... porque tengo... eh... mi abuelo tiene un campo (D, hombre, P. Subv.TP).

Campo sí... pero en otra cosa no, en oficina estar sentado, no (D, hombre, P. Subv.TP).

"Yo acá llegué como a terminar mi básica nomás":

Estructura narrativa de penitencia

En esta estructura el protagonista muere simbólicamente, encontrándose en un lugar indeseado purgando sus faltas hasta expiarlas, tras lo cual será conducido al paraíso (Gallardo, 2012). Tal estructura es identificable en los relatos de $\mathrm{J}$ y H.

En el relato de $\mathrm{J}$, el protagonista se ve obligado a acatar la voluntad de su padre respecto de permanecer en el liceo agrícola tras finalizar su enseñanza básica, implicando un duelo respecto a sus propios deseos e iniciando de esta manera su penitencia:

O sea, yo acá... llegué como a terminar mi básica nomás... y como que después me iba a cambiar (...) después me quedé... porque... no sé... como que mi papá me dijo "quédate" y yo le dije "ah, ya" (J, hombre, P. Subv.TP).

prueba siguiente; la prueba principal, donde mediante acciones heroicas el protagonista consigue su objetivo; y finalmente la prueba glorificante, en que el héroe abandona el espacio heroico, restituye la normalidad, es reconocido y recibe la recompensa prometida. 
El establecimiento en que $\mathrm{J}$ se encuentra actualmente no representa sus intereses, lo que incide en su motivación y resultados académicos:

Es que igual la materia no me gusta mucho así, como que no me interesa (J, hombre, P. Subv. TP).

Regresar al campo es significado negativamente por $\mathrm{J}$, siendo su expectativa permanecer estudiando y eventualmente trabajando en la ciudad:

Si igual, he pensado vivir acá... en Osorno para no... para que no me cueste trabajar, como pa'viajar así... y... tener un poco de movilización acá (J, hombre, P. Subv.TP).

Espera trasladarse pronto al liceo que imparte la especialidad a la cual desea ingresar. Esta decisión de cambio de establecimiento se ve claramente influenciada por sus compañeros de internado:

Sí, igual me dicen que uno se puede ir a dual, que es la empresa, que tiene una semana en el liceo y la otra semana trabajando $(\mathrm{J}$, hombre, $\mathrm{P}$. Subv.TP).

Por su parte, en su relato $\mathrm{H}$ se encuentra obligado a ingresar a la especialidad de contabilidad por mandato de su madre:

Después me vine pa'acá, a estudiar segundo (...) de ahí escogí contabilidad... aunque no me gustaba mucho pero... mi mamá... me obligó (H, hombre, Municipal TP).

A pesar de que cursar esta especialidad implica prestigio y es valorado al interior del liceo, $\mathrm{H}$ la experimenta como alejada de sus intereses:

La especialidad de "conta" [contabilidad] no me gusta porque mucha computación, y a mí no me gusta computación, me aburro... yo quería estudiar agronomía $(\mathrm{H}$, hombre, Municipal TP).

La penitencia podría extenderse indefinidamente en el tiempo y esto $\mathrm{H}$ lo vincula a ejercer profesionalmente la especialidad:

Es que no me gusta estar así como sentado... no. Me gusta estar así, estudiar algo que... estudiar algo que tenga que moverme para hartos lados $(\mathrm{H}$, hombre, Municipal TP).

Su objeto de búsqueda es terminar enseñanza media y dedicarse a una actividad que le permita viajar e interactuar socialmente. En ese contexto, una expectativa importante se vincula a regresar a su comuna de origen para desarrollar junto a su hermano un emprendimiento turístico: 
Allá tenemos nosotros como para trabajar con turismo... $(\mathrm{H}$, hombre, Municipal TP).

\section{Grados de articulación de sentido identitario}

"Estoy en clases y quiero tomar atención": Articulación de sentido identitario fuerte

Es posible identificar esta modalidad en cuatro de los seis relatos analizados, caracterizándose por un relato que presenta clara coherencia entre las metas de las acciones de tipo académico y el motivo o móvil de la actividad identitaria. En la narración de $Z$ es posible identificar nítidamente esta modalidad, ya que su ingreso al espacio heroico (liceo) define una serie de acciones relacionadas a su práctica como estudiante cuyas metas no se limitan solo al ámbito académico, sino que lo superan para proyectarse en el móvil de la actividad identitaria que, de acuerdo a su narración, sería retribuir a su madre a través de dedicarle la obtención de un título profesional:

Yo sé que para ella, esto no es un favor... no es una obligación, es algo como que ella quiere hacer porque somos sus hijas, y bueno, aunque seamos sus hijas, yo igual quiero recompensarla con algo $(Z$, mujer, Municipal HC).

Se hace evidente que el móvil de la actividad identitaria (retribuir a su madre) opera movilizando las acciones, permitiendo que el objeto de búsqueda principal de la acción de formación (ser profesional) se vincule de modo sustancial con éste, lo cual aumenta la probabilidad de involucramiento en la actividad de formación. Es posible interpretar algunas de sus acciones (como su decisión de cambiarse de puesto dentro de la sala con el objetivo de mejorar sus posibilidades de atender al profesor, distanciándose con ello de una amiga con quien compartía asiento) precisamente en este sentido:

Yo antes me sentaba con mi compañera... y ella me hablaba todo el día en la clase (...) no me daba el valor como para decirle "estoy en clases y quiero tomar atención” ... Y si yo me sentaba con ella allá atrás, no iba a entender nada. Entonces yo preferí sentarme allá adelante y sola (...) Y ahora estoy súper bien... o sea... de hecho entiendo las cosas y me siento con una niña que es... que no le cuesta... y cuando hacemos actividades yo las hago con ella y... yo aprendo, y ella aprende ( $Z$, mujer, Municipal HC).

La construcción de una imagen de sí misma como "alguien que no le cuesta" se alinea entonces tanto con la meta de la acción formativa (ser profesional) como con el objeto de búsqueda principal, retribuir a su madre. Los relatos de $\mathrm{C}$ y $\mathrm{D}$ se presentan de forma similar. En el relato de $D$ resulta notable el involucramiento con la actividad académica:

Llego a mi casa antes de acostarme, tomo el libro, repaso harto una o dos veces y al otro día antes me levanto y en el furgón voy estudiando 
un rato, llego acá en la mañana y estudio o antes de hacer la prueba igual estudio ( $D$, hombre, P. Subv.TP).

Esto puede ser entendido como una expresión del sentido que cobra su aprendizaje en términos de valor atribuido al estudio, probablemente debido al establecimiento de una conexión significativa con su experiencia cotidiana:

Es que matemáticas es difícil pero el problema es que uno a futuro aprende las fórmulas para llegar a hacer otras fórmulas... por ejemplo hoy en día uno pa' comprar un campo necesita una fórmula, cuánto mide esto cuando lo multiplico... (D, hombre, P. Subv.TP).

De forma similar, el relato de C presenta una concordancia básica entre las metas de sus acciones de formación (ser profesional) que tienden a confluir y proyectarse en el móvil de su actividad identitaria (ser independiente económicamente):

[Quiero terminar] para que mi mamá no pague todo, estudios acá, allá igual las cuotas, no quiero seguir siendo una carga más (C, mujer, Municipal TP).

\section{"Estoy aquí solo para terminar mi cuarto":8}

Articulación de sentido identitario débil

Esta modalidad se aprecia en el relato de $\mathrm{H}$, donde es posible observar que los eventos ligados a la escolarización y a la formación no guardan necesaria relación con el móvil de la actividad identitaria (en este caso, lograr la independencia económica a través de una ocupación que le permita viajar). Aquí estudiar una especialidad no deseada y pasar de curso es significado como requisito obligado para la obtención de licencia media, tras lo cual le sería posible volver a su lugar de origen en el campo para trabajar en turismo junto a su hermano y acercarse al móvil de su actividad identitaria:

[Con mi hermano en el campo] hacemos caza, pesca, cabalgata y paseos culturales... nosotros los llevamos a sectores así que por ejemplo tienen cultivo, tienen donde hacían harina antes, la gente ve el cultivo, las viejitas... ahí ayudo yo a hacer asados, a conocer lugares $(\mathrm{H}$, hombre, Municipal TP).

Estoy aquí solo para terminar mi cuarto... voy a terminar mi cuarto" $(\mathrm{H}$, hombre, Municipal TP).

Por su parte, en el relato de $\mathrm{J}$ se evidencia cómo el protagonista logra generar sentido respecto del espacio de formación, pero no con la formación en sí:

\footnotetext{
${ }^{8}$ El término "cuarto" se refiere a "cuarto medio", denominación con que en Chile se designa coloquialmente el nivel terminal de escolarización secundaria.
} 
No hace mucho hice un campeonato [de fútbol]... y ganamos primer lugar (...) entonces son como momentos de felicidad que te da la escuela, más que nada, más que la materia (J, hombre, P. Subv.TP).

Como que todos quieren divertirse nomás, como que buscan más diversión que estudios (J, hombre, P. Subv.TP).

La dimensión académica de su formación es rechazada explícitamente, apareciendo escindida respecto del objeto de búsqueda identitario (en este caso, ser mecánico):

Lo único que quiero estudiar así es como mecánica... y como que lo otro, chao (J, hombre, P. Subv.TP).

\section{Resultados emergentes}

Producto del análisis intercasos es posible identificar una serie de tópicos que cruzan la mayor parte de los relatos estudiados y que resulta necesario sintetizar a continuación, ya que forman parte importante de los sentidos que los participantes atribuyen a su actividad académica:

\section{Las figuras parentales: autoritarias o movilizadoras de sentido}

En los relatos se evidencian posiciones diferenciales de tales figuras, asociadas a diferencias en cuanto a las articulaciones de sentido identitario que los participantes presentan. En general se evidencian dos situaciones: a) la gratitud y sentimiento de deuda hacia las figuras parentales o familiares; y b) la distancia respecto de tal(es) figura(s) en el contexto de relaciones que podrían ser calificadas como autoritarias. En el primer caso - todos relatos heroicos y de articulación fuerte- la madre o abuela son representadas como los principales destinadores de sus objetos de búsqueda, en función de que son vistas como modelos de esfuerzo, sacrificio y apoyo:

Según ella eso nos va a hacer feliz [obtener un título profesional] y yo sé que sí... uno va a ser feliz si tiene estudios, tiene carrera, una profesión y tiene su trabajo (Z, mujer, Municipal $\mathrm{HC}$ ).

El sentido de gratitud hacia la familia parece inscribirse en relaciones más afectuosas, horizontales y democráticas, que a su vez parecen vincularse a la disponibilidad por parte de los jóvenes de espacios de autonomía en la toma de decisiones. En este caso, frente al ámbito vocacional:

De ahí postulé a la Industrial y de ahí en junio me trasladé aquí. Me trasladé yo solo (...) por mis propias decisiones (D, hombre, P. Subv.TP). 
Es que, bueno, yo salí de octavo allá, y le dije a mi mamá que me quería venir acá ( $\mathrm{C}$, mujer, Municipal TP).

Mis papás siempre me preguntan "mira yo tengo estas opciones" dice mi papá, por ejemplo (...) dice mi mamá “¿tú qué piensas?" (S, mujer, Municipal HC).

En contraste, relatos como los de $\mathrm{H}$ y $\mathrm{J}$-ambos de penitencia y articulación débilmuestran relaciones paterno-filiales que, al menos en lo que concierne a la elección de establecimiento, aparecen como autoritarias:

Es que mi mamá quiere que hagamos lo que ella quiere... con mis otros hermanos ha pasado lo mismo. A mi hermano le gustaba el turismo y ella le dijo que no. Cuando lo matricularon (...) le dijeron que ingeniería en administración de empresas y marketing ( $\mathrm{H}$, hombre, Municipal TP).

\section{Transición como hito en el desarrollo}

La transición al liceo significó para la casi totalidad de los participantes dejar su hogar, para vivir durante la semana en los internados de los establecimientos (cuatro casos), en casa de familiares (un caso) o viajando diariamente a su hogar en el campo (un caso). Esta transición se realiza en un rango de edad de 12 a 14 años aproximadamente, y de forma regular se asocia a un cambio importante a nivel personal, familiar y social, el que puede ser experimentado como difícil o amenazante:

Sí, me costó mucho adaptarme al liceo (...) el cambio fue tremendo porque allá aunque caminaba dos kilómetros todos los días llegaba a mi casa, en cambio acá estoy en el internado (C, mujer, Municipal TP).

Tal cambio puede llegar a tener consecuencias académicas:

El primer mes fue súper complicado porque lloraba mucho... pasaba enferma por mi misma situación psicológica (...) entonces dejé mucho de lado mis estudios por lo mismo, por extrañar y por no acostumbrarme al sistema (S, mujer, Municipal HC).

Bajé harto el promedio que tenía allá [rural] con el de acá (J, hombre, P. Subv.TP).

Estas dificultades académicas o de adaptación son significadas de modo diverso en el contexto de los relatos de los participantes. En los relatos heroicos de articulación de sentido fuerte, tales eventos tienden a experimentarse como dificultades propias de un tránsito que no resulta sencillo pero que cobra pleno sentido en su superación. En tanto, en aquellos relatos de penitencia con articulación de sentido débil, estas situaciones parecen operar como demostraciones del desajuste entre la actividad académica y el móvil identitario. 


\section{Migración al espacio urbano y retorno al espacio rural}

En la mayoría de sus relatos los participantes consideran el espacio urbano como espacio de desarrollo futuro, esto es, como el lugar en que esperan vivir, continuar estudios o trabajar, descartando el ámbito rural, debido fundamentalmente a la dificultad en el acceso a servicios disponibles más fácilmente en la zona urbana (transporte, ocio, estudio y trabajo principalmente). Resulta interesante observar sin embargo dos excepciones (relatos de $\mathrm{H}$ y D), en que la valoración positiva del lugar de origen rural se prolonga hasta convertirse en parte de la actividad identitaria, bajo la forma de objeto de búsqueda o lugar deseado, conformando una suerte de imaginario de retorno al lugar de origen. En el contexto de un relato de penitencia con articulación de sentido débil, el sujeto $\mathrm{H}$ plantea su propósito de desarrollar un emprendimiento turístico familiar en su lugar de origen, empleando los conocimientos de la especialidad a la cual espera integrarse:

Me gustaría volver ahí, o sea yo igual ayudo a mi hermano, en la caza, los paseos culturales ( $\mathrm{H}$, hombre, Municipal TP).

En la misma dirección, aunque a través de un relato heroico con articulación de sentido fuerte, se hace evidente en el sujeto D la valoración del espacio rural:

[En el campo existe] más libertad, no te molesta nadie, no hay bullicio de los autos, perros, nada (D, hombre, P. Subv.TP).

Esto contribuye a definir el sentido que le puede dar a la formación como técnico agrícola, que incluye la idea de regresar al campo para ejercer lo aprendido:

Y a mí me gustaría estudiar técnico agrícola administrativo, o sea, volver y administrar un campo así (D, hombre, P. Subv.TP).

\section{Continuación de estudios superiores}

El seguir estudios superiores -entendidos como aquellos que permiten la especialización en algún área específica, ya sea de tipo técnico o profesional (centros de formación técnica, institutos profesionales y universidades) - constituyó un tema recurrente en la totalidad de los relatos, adquiriendo una valoración altamente positiva evidenciable en la identificación con las metas de la acción de formación, e incluso con el motivo de la actividad identitaria:

Que vayamos a la universidad o instituto y que consigamos un título con sudor y esfuerzo (Z, mujer, Municipal HC).

En los relatos de los participantes seguir estudios superiores se constituye en objeto de búsqueda estructurante que configura el sentido de la transición al espacio urbano. 


\section{DISCUSIÓN}

Los resultados expuestos permiten caracterizar distintas formas en que estudiantes de origen rural que realizan estudios medios en liceos urbanos otorgan sentido a su actividad académica, por medio de la exploración de la estructura narrativa de sus relatos de práctica. En estas narraciones fue posible identificar dos modalidades de articulación que se diferencian de acuerdo con el grado de concordancia entre la meta de las acciones de formación y el móvil identitario, apareciendo asociadas a estructuraciones narrativas reconocibles en estudios anteriores.

En convergencia con estudios previos que emplean similar perspectiva de análisis (Sebastián y De Villers, 2006; Gallardo, 2012; Orrego y Sebastián, 2012), destaca la relación que fue posible establecer entre la estructura heroica y una modalidad de articulación de sentido fuerte. Esto sugiere que la presencia de un destinador -quien realiza el contrato con el protagonista lanzándolo al espacio heroico en demanda de su objeto de búsqueda (principalmente en esta investigación, figuras como la madre o la abuela) - parece resultar fundamental para la construcción progresiva por parte del sujeto de un sólido sentido identitario en torno a su actividad como estudiante, que podría facilitar un mayor involucramiento con el propio proceso de aprendizaje (Bourgeois, 2009).

En los casos de los participantes que muestran sentido identitario fuerte, tales figuras familiares -además de operar como destinadores - parecen permitir que el protagonista se construya a sí mismo como agente en relación con decisiones vinculadas a la continuación de estudios medios — con posibilidad de expresar sus intereses y por lo tanto influir frente a su familia-, situación que contrasta notablemente con los relatos del tipo "penitencia". Efectivamente, en estos últimos el protagonista aparece sin posibilidad de expresar su voluntad, o expresándola en vano, frente a figuras parentales centradas en lo normativo, sometiéndose por ende a decisiones que le conciernen directamente pero que no incluyen sus propios puntos de vista. En estas condiciones el estudiante puede desarrollar un sentido de obligación respecto de la formación (Tenti, 2000), una articulación de sentido débil, ya que las metas de sus acciones cotidianas en el espacio educativo presentan escasa o nula vinculación con el móvil identitario del sujeto.

Tal situación contribuiría a configurar una forma de exclusión educativa que Terigi (2009) denomina "escolaridad de baja intensidad": los estudiantes si bien pueden desarrollar involucramiento con el espacio de formación (socialización, amistades, participación en actividades extraacadémicas), no logran involucrarse con la formación en sí. Desde luego, con lo anterior no se pretende afirmar que el carácter de las transiciones educativas dependa exclusivamente de tales condicionantes. Sabemos que estas transiciones más bien se inscriben en las trayectorias educativas y sociales de los sujetos, expresando el encuentro entre elecciones propias de los jóvenes, 
trayectorias familiares y oferta de las instituciones disponibles (Montes y Sendón, 2006; Crivello, 2009).

Es precisamente esta evidencia la que parece sugerir que un mejor soporte de las transiciones educativas de los jóvenes desde espacios rurales a urbanos depende de la provisión, por parte de los establecimientos básicos y secundarios, de apoyos adecuados, en términos de - por ejemplo- considerar al momento de la postulación al establecimiento secundario los determinantes de la decisión familiar e individual que definen su elección, el grado de conocimiento de la oferta educativa, así como la consistencia entre esta elección y las inquietudes vocacionales del estudiante. Lo anterior requiere el diseño de políticas que promuevan en los establecimientos secundarios la necesidad de generar criterios y dispositivos que aseguren la pertinencia de la oferta educativa, así como el diseño de trayectorias formativas más flexibles que favorezcan la construcción de narrativas de sentido identitario fuerte en los estudiantes.

Con independencia del tipo de estructura narrativa, todos los relatos de los jóvenes participantes de esta investigación reflejan el alto valor asignado por las familias rurales a la educación como forma de desarrollo y promoción social, lo cual es consistente con estudios recientes en Chile y Latinoamérica (Hernández y Raczynski, 2014; Ames y Rojas, 2011; Silva-Peña et al., 2013). En este sentido, la expectativa de continuar estudios superiores —y por lo tanto salir del espacio rural- es para todos los jóvenes participantes de esta investigación un elemento estructurante de sus relatos identitarios.

Esto puede vincularse con el extendido discurso de padres y docentes acerca de seguir estudios superiores como fórmula de éxito social (Rozas y Lara, 2009; SilvaPeña et al., 2013). Sin embargo, resulta interesante observar un movimiento distinto en dos relatos ( $\mathrm{H}$ y $\mathrm{D}$ ) que - a diferencia de los anteriores - sitúan su móvil identitario en el retorno a la zona rural de procedencia, y que correspondería a una lógica asimilable a la migración de retorno: aquella acción migratoria que implica un regreso posterior al lugar de origen (Organización Internacional del Trabajo, 2012). Es posible relacionar este fenómeno con las profundas y aceleradas transformaciones que ha sufrido el espacio rural en el último medio siglo (Ascorra, 2012), que autorizan a referir como "nueva" a una ruralidad que actualmente presenta mayor diversificación de la estructura productiva y mayor y más compleja integración con el espacio urbano, haciéndose por lo tanto crecientemente atractiva como espacio de residencia y disfrute (Borsdorf y Hidalgo, 2009). Resulta plausible suponer que tal cambio en la representación social de lo rural se manifieste en las estructuras narrativas empleadas por los jóvenes rurales, a través de la presencia de elementos como el del retorno.

Finalmente, los resultados de este estudio destacan la importancia de la construcción de sentido de la formación por parte de los estudiantes con el propósito de favorecer su motivación e involucramiento con el aprendizaje, lo que parece particularmente necesario en el contexto de la crisis de los sistemas educativos. 
Pensamos que aspectos derivados, como el diseño de trayectorias formativas o la exploración de formatos de trabajo y prácticas (de admisión, de instrucción, evaluativas, entre otras) que ofrezcan la oportunidad a los jóvenes de construir sentido en torno a su actividad académica, representan tareas necesarias de abordar por futuras investigaciones.

\section{REFERENCIAS}

Alvarado, S., y Suárez, M. (2009). Las transiciones escolares: una oportunidad de desarrollo integral para niños y niñas. Revista Latinoamericana de Ciencias Sociales, Niñez y Juventud, 7(2), 907-928.

Ames, P., y Rojas, V. (2011). Cambios y oportunidades: La transición de la escuela primaria a la secundaria en el Perú. Recuperado de http://www.ninosdelmilenio.org/wpcontent/uploads/2012/10/dt63.pdf

Agencia de Calidad de la Educación. (2012). Metodología de construcción de grupos socioeconómicos. Pruebas SIMCE 2012. Recuperado de http://archivos.agenciaeducacion.cl/Metodologia-deConstruccion-de-Grupos-Socioeconomicos-SIMCE-2012.pdf

Arciero, G. (2003). Estudios y diálogos sobre la identidad personal. Reflexiones sobre la experiencia humana. Buenos Aires, Argentina: Amorrortu.

Ascorra, P. (2012). Ruralidad: Desafíos y proyecciones para los estudios sociales. Psicoperspectivas 11(1), 1-7. https://doi.org/10.4067/S0718-69242012000100001

Baeza, J. (2001). El oficio de ser alumno, en jóvenes de liceo de sector popular. Santiago, Chile: Ediciones Universidad Católica Cardenal Raúl Silva Henríquez.

Bolívar, A., Domingo, J., y Fernández, M. (1998). La investigación biográfica narrativa en educación. Granada, España: Universidad de Granada/Force.

Borsdorf, A., y Hidalgo, R. (2009). Searching for Fresh Air, Tranquillity and Rural Culture in the Mountains: A New Lifestyle for Chileans?. Journal of the geographical society of Berlin, 140(3), 275-292.

Bourgeois, E. (2009). Apprentissage et transformation du sujet en formation. En J.M. Barbier, E. Bourgeois, G. Chapelle, y J.-C. Ruano-Borbalan (Dirs.), Encyclopédie de la formation (pp. 31-71). París, Francia: PUF.

Crivello, G. (2009). "Becoming somebody": Youth transitions through education and migration-evidence from Young Lives, Perú. Recuperado de https://www.younglives.org.uk/sites/www.younglives.org.uk/files/YL-WP43-CrivelloBecomingSomebody.pdf

Eisenhardt, K. (1989). Building Theories from Case Study Research. Academy of Management Review, 14(4), 532-550. https://doi.org/10.2307/258557

Elige Educar. (2016). Radiografía de la educación rural en Chile. Recuperado de http://eligeeducar.cl/radiografia-de-la-educacion-rural-en-chile 
Espínola, V., Claro, J. P., y Walker, H. (2009). Lineamientos estratégicos para la discusión de una política de mediano plazo para la educación media. Reporte de investigación. Recuperado de https://es.scribd.com/document/82099129/Lineamientos-Educacion-Media-Diagnostico

Flick, U. (2004). Introducción a la investigación cualitativa. Madrid, España: Morata/Fundación Paideia Galiza.

Gallardo, G. (2012). Articulaciones de sentido identitario con la formación construidas por jóvenes estudiantes secundarios en dos liceos de nivel socioeconómico medio bajo de la Región Metropolitana. Un contraste desde el rendimiento SIMCE. (Tesis de Maestría). Pontificia Universidad Católica de Chile, Santiago, Chile.

Gallardo, G., y Sebastián, C. (2012). Anexo metodológico. En G. Gallardo, Articulaciones de sentido identitario con la formación construidas por jóvenes estudiantes secundarios en dos liceos de nivel socioeconómico medio bajo de la Región Metropolitana. Un contraste desde el rendimiento SIMCE. (Tesis de Maestría). Pontificia Universidad Católica de Chile, Santiago, Chile.

Gallardo, G., y Sebastián, C. (2015). Exclusión e involucramiento con el aprendizaje: una propuesta de comprensión desde los enfoques socioculturales. En P. Freire, R. Moretti y F. Burrows (Eds.), Aprender con otros. Aproximaciones psicosociales sobre el aprendizaje en contextos educativos. Santiago, Chile: Ediciones Universidad Alberto Hurtado.

Galton, M. (2009). Moving to secondary school: initial encounters and their effects. Perspectives on Education, 2, 5-21.

Gimeno Sacristán, J. (1997). La transición a la Educación Secundaria. Discontinuidades en las culturas escolares. Madrid, España: Morata.

Glassman, M. (1996). Understanding Vygotsky's motive and goal: An exploration of the workof A.N. Leontiev. Human Development, 39, 309-327. https://doi.org/10.1159/000278505

Greimas, A. (1966). Semántica estructural. Madrid, España: Gredos.

Greimas, J. (1989). Del sentido. Madrid, España: Gredos.

Hernández, M., y Raczynski, D. (2014). Jóvenes de origen rural: aspiraciones y tensiones en la transición hacia la enseñanza secundaria. Revista Iberoamericana de Evaluación Educativa, 7(3), 71-87.

Howard, S., y Johnson, B. (2004). Transition from primary to secondary school: possibilities and paradoxes. Ponencia presentada en la International Education Research Conference, Australian Association for Research in Education, Melbourne, Australia. Recuperado de https://www.aare.edu.au/publications-database.php/4264/transition-from-primary-to-secondaryschool-possibilities-and-paradoxes

Instituto Nacional de Estadísticas de Chile. (2010). Anuario Estadístico 2008. Región de Los Lagos. Puerto Montt, Chile: Instituto Nacional de Estadísticas de Chile.

Instituto Nacional de la Juventud de Chile. (2015). Informe Octava Encuesta Nacional de Juventud. Santiago, Chile: Instituto Nacional de la Juventud de Chile.

Johnstone, K. (2002). The transition to high school: a journey of uncertainty. Ponencia presentada en la International Education Research Conference, Australian Association for Research in Education, 
Brisbane, Australia. Recuperado de https://www.aare.edu.au/publications-database.php/3511/thetransition-to-high-school-a-journey-of-uncertainty

Leontiev, A. N. (1984). Actividad, conciencia y personalidad. México D.F., México: Cártago.

Leyton, T. (2013). Las políticas de educación rural en Chile: cambio y continuidad. Recuperado de http://actacientifica.servicioit.cl/biblioteca/gt/GT5/GT5_LeytonM.pdf

Ministerio de Educación de Chile. (2011). Informe final de evaluación Programa Pro-retención. Santiago, Chile: Ministerio de Educación de Chile.

Ministerio de Educación de Chile. (2013a). Medición de la deserción escolar en Chile. Serie Evidencias, Centro de Estudios MINEDUC. Santiago, Chile: Ministerio de Educación de Chile.

Ministerio de Educación de Chile. (2013b). Estadísticas de la Educación 2013. Centro de Estudios MINEDUC. Santiago, Chile: Ministerio de Educación de Chile.

Montes, N., y Sendón, M. (2006). Trayectorias educativas de estudiantes de nivel medio. Argentina a comienzos del siglo XXI. Revista Mexicana de Investigación Educativa, 11(29), 381-402.

Nuñez, C., Solís, C., y Soto, R. (2014). ¿Qué sucede en las comunidades cuando se cierra la escuela rural? Un análisis psicosocial de la política de cierre de las escuelas rurales en Chile. Universitas Psychologica, 13(2), 615-625.

Organización Internacinal del Trabajo. (2012). Migración Laboral Internacional: un enfoque basado en los Derechos. Madrid, España: Plaza y Valdés.

Orrego, K., y Sebastián, C. (2012). Construcción de sentido identitario en narrativas de experiencias de alfabetización en adultos chilenos. (Manuscrito no publicado).

Patton, M. (1991). Qualitative Evaluation and research methods. Londres, Inglaterra: Sage.

Penuel, W., y Wertsch, J. (1995). Vygotsky and identity formation: a sociocultural approach. Educational Psychologist, 30, 83-92. https:// doi.org/10.1207/s15326985ep3002_5

Pereira, A., y Pooley, J. (2007). A qualitative exploration of the transition experience of students from a high school to a senior high school in rural Western Australia. Australian Journal of Education, 51, 162-177. https://doi.org/10.1177/000494410705100205

Pietarinen, J. (septiembre de 1998). Rural school students' experiences on the transition from primary school to secondary school. Presentado al European Conference for Educational Research, University of Ljubljana, Slovenia.

Raczynski, D. (2011). El paso de la enseñanza básica a la media en estratos bajos: Un reto a la igualdad de oportunidades educativas. Informe de investigación. Recuperado de http://www.comunidadescolar.cl/documentacion/FONIDE/Informe\%20Final-Dagmar\%20RaczynskiF511083.pdf

Rice, J. (2001). Explaining the negative impact of the transition from middle to high school on student performance in mathematics and science. Educational Administration Quarterl, 37(3), 372-400.

Rozas, C., y Lara, C. (2009). Jóvenes rurales y educación media: un estudio exploratorio acerca de la relación entre jóvenes rurales escolarizados de la provincia de Cachapoal, región de O'Higgins y la educación media en Chile. En J. Redondo y L. Muñoz (Eds.), Juventud y enseñanza media en 
Chile del bicentenario. Antecedentes de la revolución pingüina (pp.128-163). Santiago, Chile: Observatorio Chileno de Políticas Educativas.

Santos, H. (2009). Dinámica de la deserción escolar en Chile. Santiago, Chile: Centro de Políticas Comparadas de Educación/Universidad Diego Portales.

Sebastián, C., y De Villers, G. (2006). Activité identitaire et Sens de la Formation. Une étudeexploratoire du point de vue histórico-culturel. En J. M. Barbier, E. Bourgeois, G. De Villers y M. Kaddouri (Eds.), Constructions identitaires et mobilisation des sujetsen formation (pp. 185-228). París, Francia: L'Harmattan.

Sebastián, C., Gallardo, G., y Calderón, M. (2016). Sentido identitario de la formación. Una propuesta para articular el desarrollo de la identidad y el aprendizaje en contextos educativos. Papeles de Trabajo sobre Cultura, Educación y Desarrollo Humano, 12(3), 4-12. Recuperado de http://psicologia.udg.edu/PTCEDH/admin/publicacions/4.pdf

Silva-Peña, I., Bastidas, K., Calfuqueo, L., Díaz, J., y Valenzuela, J. (2013). Sentido de la escuela para niños y niñas mapuche en una zona rural. Polis, 12(34), 243-258.

Tenti, E. (2000). Culturas juveniles y Cultura Escolar. Buenos Aires, Argentina: IIPE-UNESCO.

Terigi, F. (2009). La inclusión educativa: viejas deudas y nuevos desafíos. En F. Terigi (Coord)., Segmentación urbana y educación en América Latina. El reto de la inclusión escolar (pp. 21-28). Madrid, España: FIECC.

Tilleczek, K., y Ferguson, B. (2007). Transitions and Pathways from Elementary to Secondary. Literature Review Report to the Ministry of Education. Sidney, Australia: Ministry of Education.

Valenzuela, J. (2009). Características psicométricas de una escala para caracterizar el sentido del aprendizaje escolar. Universitas Psychologica, 8(1), 49-59.

Vera-Bachmann, D., y López, M. (2014). Resiliencia Académica: una alternativa a explorar en la educación de niños y niñas en contextos rurales. Integra Educativa, 7(2), 187-205.

Vera-Bachmann, D. (2015). Resiliencia, Pobreza y Ruralidad. Revista Médica de Chile, 143(5), 677-678.

Vera-Bachmann, D., y Salvo, S. (2016). Perfiles de Escuelas Rurales exitosas. Una propuesta de la psicología educacional ante el cierre de escuelas. Universitas Psychologica, 15(2), 15-28.

Walsh, M. (1995). Rural students' transitions to secondary school: culture, curriculum and context. The Curriculum Journal, 6(1), 115-127. https://doi.org/10.1080/0958517950060108

Wertsch, J. (1993). Voces de la mente. Madrid, España: Visor.

West, P., Sweeting, H., y Young, R. (2010). Transition matters: pupils' experiences of the primarysecondary school transition in the West of Scotland and consequences for well-being and attainment. Research Papers in Education, 25(1), 21-50.

Yin, R. (1994). Case Study Research. Design and Methods, Applied Social Research Methods (Vol. 5, 2 ed.). Newbury Park, CA: Sage.

Zapata, S. (2000). Al encuentro del joven rural. Santiago, Chile: Agencia de Cooperación IICA Chile. 